\title{
Transduction and Segregation in Escherichia coli K12
}

\author{
Alian CampBeLL \\ Department of Barteriology, University of Michigan, Ann Arbor, Michigan
}

Accepted June $21,195 \%$

\begin{abstract}
The transduction of the gal ${ }^{+}$character into gal mutants of Escherichia coli K12 with high frequency transducing (HFT) lysates of phage $\lambda$ has been studied. Sensitive recipients infected at low multiplicity give rise to heterogenotes carrying a defective prophage. Simultaneous infection with a nontransducing phage increases the probability of transduction and causes the cell to become lysogenic. Ifysogenic recipients upon transduction generally remain lysogenic for the original prophage. Prophage substitution and double lysogenization rarely occur. I,oss of the gal ${ }^{+}$character from the defective lysogenics is generally accompanied by loss of the defective prophage. Loss of the gal ${ }^{+}$character from the double lysogenies is correlated with changes in prophage content.
\end{abstract}

\section{INTRODUCTION}

Morse et al. (1956a) discovered that coliphage $\lambda$ is able to transduce a group of linked genetic markers controlling galactose utilization. In the original experiments, gal ${ }^{+}$donors and gal $^{-}$recipients were used. Under these conditions, the descendants of most of the transduced cells have two very interesting properties: (1) They are unstable, and spontaneously lose the galt character with a frequency of about $10^{-3}$ per bacterium per generation. The gal- segregants are negative at the same locus within the region as was the original strain before transduction; showing that there was an addition to, rather than a replacement of a portion of, the bacterial genome. The unstable strains have been named heterogenotes. (2) Lysates produced by induction of heterogenotes will transduce sensitive strains with high frequency, approaching in some lysates one transduction per placue-forming unit (high frequency transduction, HFT).

The block of galactose genes which enter the cell by transduction c:an be called the exogenes (Morse et al., 1956b).

${ }^{1}$ Supported by United States Public Health Grant E-1205. The proliminary experiments were performed at the California Institute of Technology. 
Morse et al. observed very low burst sizes when heterogenotes were induced. Weigle (1957), using a different strain of $\lambda$, obtained burst sizes above 100 . This allowed Weigle to demonstrate that the exogenes very probably multiply after induction, because each lysing cell produces, on the average, several transducing particles. He also showed hat the number of transducing particles increases during a cycle of lytic growth. Both observations suggest that the phage genetic material and the exogenes are physically attached in the heterogenote. ${ }^{2}$

The present investigation is an attempt to clarify further the relationship between the phage and the transduced genes.

\section{MATERIALS AND METHODS}

\section{Bacteria}

All are derivatives of $E$. coli K12 obtained from the collection of Dr. $J$. Weigle. The gal ${ }^{-} \lambda$-sensitive strain used for most of the experiments was Wollman's (1953) strain 112. In later work, where spontaneous reversions to $\mathrm{gal}^{+}$became a serious technical difficulty, a gal $_{1} \mathrm{gall}_{2}^{--}$ strain produced by Dr. Weigle from strains of Morse et al. (1956b) was used. It had been isolated as a $\lambda$-sensitive survivor from ultraviolet irradiation of a gal- segregant from a heterogenote produced by transduction of Lederberg's strain W3101 ( $\mathrm{gal}_{1}^{-}$) with a lysate from W3102 (gal - homogenote). Phage were plated on Appleyard's (1956) strains C600 and CR63. CR63 does not adsorb wild type $\lambda$ and is an indicator for the $\mathrm{h}$ marker.

\section{Phages}

Some early experiments were done with wild type $\lambda$ and $\lambda \mathrm{h}$ (Appleyard et al., 1956) obtained from Dr. Weigle. For most of the work, reference type $\lambda$ and $\lambda \mathrm{m}_{5} \mathrm{CO}_{2}{ }^{2 a}$ (Kaiser, 1955), were used. The mutant $\lambda \mathrm{h}$ was prepared by plating on CR63 an induced lysate of a derivative of C600 which carried $\lambda$ reference type, and purifying the resulting plaques by several serial replatings on CR63. Two types of such h mutants were obtained, which differed in that one formed poor plaques on CR63. They appeared alike on $\mathrm{C} 600$ or on mixed indicator. Crosses indicated that the mutations were allelic and were located between the genes $m_{5}$ (small plaque type) and $\mathrm{Co}_{2}$ (clear plaque type, with central ring of sec-

${ }^{2}$ In the present investigation, no difference in titer of induced lysates has been observed between heterogenotes and other lysogenic strains.

2a They were kindly furnished by Dr. A. J. Kaiser. 
ondary growth), as is the $\mathrm{h}$ of $\Lambda$ ppleyard ( $\Lambda$ ppleyard et al., 1956; Kaiser, 1955). The region $m_{5}-c_{2}$ comprises slightly less than $1 / 2$ the known linkage map of $\lambda$.

Lambda $\mathrm{h}$ HFT was prepared by first lysogenizing CR63 (which is gal $^{+}$) with $\lambda \mathrm{h}$. Phage from an induced lysate (low frequency transducing, LFT) were mixed with cells of bacterium 112 and plated. As expected from the results of Morse et al. (1956a), most of the gal' papillae from such plates were heterogenotes, induced lysates from which transduced with high frequency.

Induced lysates were prepared by irradiating lysogenic cells in buffer: $\mathrm{KH}_{2} \mathrm{PO}_{4}-2 \mathrm{~g} / 1 ; \mathrm{K}_{2} \mathrm{HPO}_{4}-7 \mathrm{~g} / 1 ; \mathrm{MgSO}_{4} \cdot 7 \mathrm{H}_{2} \mathrm{O}-0.25 \mathrm{~g} / \mathrm{l}$. One part of irradiated suspension was added to one part tryptone broth supplemented with $0.025 \% \mathrm{MgSO}_{4} \cdot 7 \mathrm{H}_{2} \mathrm{O}$ at $37^{\circ}$. The culture was aerated for $90-110$ minutes, by which time lysis was generally apparent. After shaking with chloroform, the lysates were centrifuged, and the supernates were stored over chloroform at $4^{\circ}$. They generally remained free from bacteria and were tested for sterility at the time of use in all critical experiments.

\section{Media}

Eosin methylene blue galactose agar (EMB) : Bacto tryptone (Difco)-$10 \mathrm{~g}$; yeast extract (Difco) $-1 \mathrm{~g} ; \mathrm{NaCl}-5 \mathrm{~g}$; galactose $-10 \mathrm{~g}$; methylene blue- $0.065 \mathrm{~g}$; eosin- $0.4 \mathrm{~g} ; \mathrm{K}_{2} \mathrm{HPO}_{4}-2 \mathrm{~g}$; deionized water -1 liter. Tryptone broth: Bacto tryptone $-10 \mathrm{~g}$; $\mathrm{NaCl}-5 \mathrm{~g}$; thiamin hydrochloride $1 \mu \mathrm{g}$; deionized water -1 liter. Tryptone agar and tryptone top agar were tryptone broth with $10 \mathrm{~g}$ and $6.5 \mathrm{~g}$ of agar per liter, respectively.

\section{Transductions}

For scoring the galactose character, streak or spread plates were made on EMB agar. When efficiency of transduction was measured as a function of multiplicity, the following procedure was used: $1 \mathrm{ml}$ of an overnight culture in tryptone broth was added to $9 \mathrm{ml}$ of tryptone broth at $37^{\circ}$ and aerated for 4 hours. It was then centrifuged and resuspended in $30 \mathrm{ml}$ chilled $0.01 M \mathrm{MgSO}_{4}$. A sample of $0.8 \mathrm{ml}$ of this suspension was added to $0.2 \mathrm{ml}$ of lysate at an appropriate dilution (the diluent being the same medium in which the lysate was prepared). It was then incubated 20 minutes at $37^{\circ}$, diluted $1: 10$ into anti- $\lambda$ serum in tryptone broth, incubated 30 minutes, diluted, and plated.

At the lowest multiplicities used, it was generally necessary to spread 
$0.05 \mathrm{ml}$ of undiluted bacteria from the serum suspension. This gives about $2 \times 10^{6}$ bacteria per plate. It was felt desirable to keep the total number per plate constant in case any positives were suppressed by competition on the dense plates; so to all plates on which diluted samples were spread, $0.05 \mathrm{ml}$ of a suspension of uninfected bacteria similarly treated was added and spread with the sample. However, control experiments showed that this made no significant difference in the counts obtained.

With the 112 strain, dense plates must be counted after 2 days incubation, since beyond that time a large number of spontaneous reversions appear. In the $\mathrm{gal}_{1}^{-} \mathrm{gal}_{2}^{-}$strain of Weigle, no spontaneous reversions appear for several days. When they do, they are very small colonies easily distinguishable from the positives produced by transduction. Counting the same plates at 2 days and at 7 days sometimes revealed an increase in the number of positive colonies by as much as $50 \%$, but the shape of the curve was not appreciably altered.

\section{Phage Scoring}

To score strains for type of carried phage, one loopful of an overnight culture was added to $1 \mathrm{ml}$ tryptone broth at $37^{\circ}$, incubated 5-8 hours, and shaken with 2 drops of chloroform. After 10-30 minutes, 0.01-0.1 $\mathrm{ml}$ was added to $0.5 \mathrm{ml}$ of plating bacteria. The latter were from 2-3-hour aerated cultures of $\mathrm{C} 600$ or CR63 in tryptone broth (inoculated with 1 $\mathrm{ml}$ of a fully grown standing culture per $10 \mathrm{ml}$ broth). Plating bacteria were centrifuged, resuspended in $0.01 \mathrm{M} \mathrm{MgSO}_{4}$, and irradiated with ultraviolet light to about $30 \%$ survival (Kaiser, 1955).

Customarily, plating was first done on a mixed indicator (1 part CR63 to $10 \mathrm{C} 600$ ), under which conditions $\mathrm{h}^{+}$plaques are speckled, and the co character is not scorable in $\mathrm{h}^{+}$plaques. If a strain carries at least one prophage with the $h$ allele, its entire content of prophage alleles can be ascertained by plating on a mixed indicator. If it does not, it must also be tested on $\mathrm{C} 600$. At least 100 plaques were examined from each culture. Absence of co $\left(\right.$ or $\left.\mathrm{co}^{+}\right)$was not inferred unless at least 100 plaques on $\mathrm{C} 600$ had been scored.

\section{RESULTS}

\section{Efficiency of Transduction as a Function of Multiplicity of Infection}

The effect of varying the multiplicity of infection of an HFT lysate on the number of transductions is shown in Fig. 1 (curve B). The ordinate is the efficiency of transduction, defined as the number of transductions 


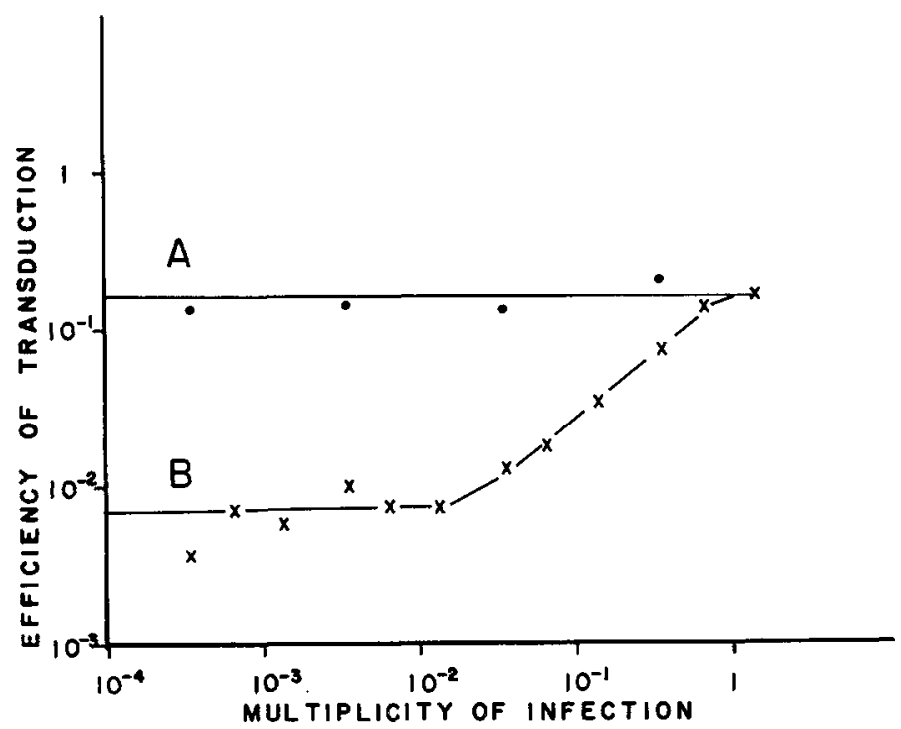

Fig. 1. Efficiency of transduction as a function of multiplicity of infection. Details in text. Curve $B=$ high frequency transducing lysate alone. Curve $A=$ same lysate with constant background multiplicity (1.7) of nontransducing phage.

per plaque-forming unit adsorbed. The curve is seen to approach two asymptotes, one for low multiplicity, another for high, separated from each other by a factor of about 20 . Table 1 shows the effect of multiplicity when lysogenic bacteria are used as recipients; curve $A$ of Fig. 1 shows the result of using a constant background multiplicity (1.7) of a nontransducing phage, produced by induction of lysogenic bacteria which were gal-. In neither of these cases does the multiplicity of transducing phage significantly affect the efficiency of transduction; the efficiencies are roughly equal to those for the lower and higher asymptotes, respectively.

Somewhat different curves have been obtained with recipient cells of different ages. There are also quantitative variations between lysates. In all cases, the sensitive recipient shows a strong multiplicity effect which is absent for lysogenic recipients, and which is removed by adding a constant multiplicity of nontransducing phage.

The decrease in transduction efficiency with decreasing multiplicity does not conflict with the results of Morse et al. (1956a), who obtained a constant efficiency over a range of multiplicities. Their method of 
TABILE 1

EFfect of Multiplicity of Infection on Efficiency of

TransDuetion

\begin{tabular}{lcc}
\hline $\begin{array}{l}\text { Multiplicity } \\
\text { HFT phage }\end{array}$ & \multicolumn{2}{c}{ Effiency of transduction } \\
\cline { 2 - 2 } 2.5 & $1.9 \times 10^{-3}$ & Sensitive recipient \\
1.0 & $1.9 \times 10^{-3}$ & $1.4 \times 10^{-1}$ \\
$2.5 \times 10^{-1}$ & $1.9 \times 10^{-3}$ & $3.3 \times 10^{-2}$ \\
$1.0 \times 10^{-1}$ & $1.6 \times 10^{-3}$ & $6.5 \times 10^{-3}$ \\
$2.5 \times 10^{-2}$ & & $2.8 \times 10^{-3}$ \\
$1.0 \times 10^{-2}$ & & \\
$2.5 \times 10^{-3}$ & & \\
$1.0 \times 10^{-3}$ & & \\
\hline
\end{tabular}

adsorbed for 20 minutes in $0.01 \mathrm{M} \mathrm{MgSO}_{4}$ at $37^{\circ}$. More than $90 \%$ of the phage adsorb. Then diluted into anti- $\lambda$ serum for 10 minutes at $37^{\circ}$, diluted further, and plated. Constant background of about $2 \times 10^{6}$ uninfected bacteria per plate.

assay (mixing phage and bacteria on plates) restricted them to very low multiplicities, at which the efficiency approaches constancy in our results also. At such low multiplicities, in their system, lysogenic recipients are transduced with a higher frequency than sensitive recipients; in ours, the two approach approximately equal values.

\section{Properties of the Transduced Clones}

Under the conditions of our experiments, the transduction clones appear as $\mathrm{gal}^{+}$papillae on plates with confluent growth. Some of these papillae have been purified by restreaking. $\mathrm{Gal}^{+}$subcolonies have been tested for lysogenicity by spot testing on $\mathrm{C} 600$, and for immunity to superinfection by cross-streaking against $\lambda$. The results are shown in Table 2 . Most of the transductions at low multiplicity give rise to strains which liberate little or no phage upon induction but which are immune to superinfection by $\lambda$ phage. We shall refer to such strains as defective lysogenics (see Appleyard, 1954). The sensitives that have occurred have all been stable rather than heterogenotic.

It is of interest to know what happens to the immunity when the defective heterogenotes lose the gal ${ }^{+}$character. Twenty-six gal ${ }^{+}$colonies coming from three different transduction clones were restreaked on galactose agar, and one gal ${ }^{+}$and one gal- subcolony from each were tested for immunity (Table 3). The great majority of the gal ${ }^{-}$segregants 
TABLE 2

Effect of Multiplicity of Infection on Constitution of Transduction Clones ${ }^{a}$

\begin{tabular}{|c|c|c|c|c|c|}
\hline \multirow{2}{*}{ Exyerintent } & \multirow{2}{*}{$\begin{array}{l}\text { Recipient } \\
\text { bacterium }\end{array}$} & \multirow{2}{*}{ Mtultiplicity } & \multicolumn{3}{|c|}{ Transduction clones } \\
\hline & & & Lysogenic & Defective & Sensitive \\
\hline 187 & $\mathrm{gal}_{1}^{-} \mathrm{gal}_{2}^{-}$ & $1.9 \times 10^{-3}$ & 1 & 22 & 2 \\
\hline \multirow[t]{4}{*}{176} & 112 & 1.6 & 19 & () & 1 \\
\hline & & $1.6 \times 10^{-1}$ & 19 & 1 & 0 \\
\hline & & $1.6 \times 10^{-2}$ & 11 & 8 & 1 \\
\hline & & $1.6 \times 10^{-3}$ & 2 & 17 & 1 \\
\hline 168 & 112 & $2.4 \times 10^{-3}$ & 1 & 14 & 5 \\
\hline
\end{tabular}

a Details as in Table 1 . Transduction clones purified by restreaking on EMB galactose agar and tested by cross-streaking and by spot testing on C600.

TABLE 3

Loss livexts from Defective Iysogenic Strains ${ }^{a}$

\begin{tabular}{ccccccc}
\hline \multicolumn{2}{c}{ Colonies from same subclone } & & \multicolumn{2}{c}{ Isolate } & Total \\
\cline { 1 - 1 } Gal $^{+}$ & $\mathrm{Gal}^{-}$ & & $\mathrm{A}$ & $\mathrm{B}$ & $\mathrm{C}$ & \\
\hline $\mathrm{I}$ & $\mathrm{S}$ & & 6 & 6 & 12 & 24 \\
$\mathrm{I}$ & $\mathrm{I}$ & & 0 & 1 & 0 & 1 \\
$\mathrm{~S}$ & $\mathrm{~S}$ & & 0 & 1 & 0 & 1 \\
\hline
\end{tabular}

${ }^{a}$ In each experiment, a $\mathrm{gal}^{+}$papilla was restreaked twice on galactose agar, several $\mathrm{gal}^{+}$colonies each restreaked from the second plate, and one positive and one negative colony from each of the final plates tested by cross-streaking. $\mathrm{I}=$ immune. $\mathrm{S}=$ sensitive

are sensitive. The very high correlation in loss suggests that the defective prophage and the exogenes remain physically attached during bacterial growth and can therefore be lost as a unit.

As has this author, Arber (personal communication) too, has observed an increased proportion of defective lysogenies when transductions are performed at very low multiplicities. With sensitive recipients, Morse et al. (1956a) obtained mostly lysogenic (rather than defective lysogenic) transduction clones when they used a multiplicity of 0.24 ; but Table 2 shows that this multiplicity is far from low enough to insure that one is dealing with the results of single infection.

\section{The Character of the Prophage in the Lysogenized Clones}

The multiplicity effert can be restated as follows: The presence in the same cell of a healthy phage element that becomes prophage increases 
TABLE 4

Prophage Constitution of Transuuction Clones Produced by Mixtures of Lysates ${ }^{a}$

\begin{tabular}{|c|c|c|c|}
\hline Nontransducing phage & Transducing phage & $\begin{array}{c}\text { Transduction } \\
\text { clones carrying } \\
\lambda \mathrm{h}\end{array}$ & $\begin{array}{l}\text { Transduction } \\
\text { clones not } \\
\text { carrying } \lambda \mathrm{h}\end{array}$ \\
\hline $\begin{array}{l}\lambda \mathrm{h} \\
\left(\mathrm{m} .0 . \mathrm{i}^{b}=3.7\right) \\
\lambda \mathrm{h}^{+} \\
(\mathrm{m} .0 . \mathrm{i} .=0.76)\end{array}$ & $\begin{array}{l}\lambda \mathrm{h}^{+} \\
\left(\mathrm{mo.i}=1.5 \times 10^{-3}\right) \\
\lambda \mathrm{h} \\
\left(\mathrm{m} . \text { o.i. }=7.5 \times 10^{-4}\right)\end{array}$ & 20 & 20 \\
\hline
\end{tabular}

a Details as in Table 1. All clones tested were lysingenic (positive spot test on $\mathrm{C} 600)$.

${ }^{b}$ m.o.i. = multiplicity of infection.

the probability that the transduced fragment will become (unstably) a part of the bacterial genome. The tests used do not distinguish whether or not the transduced fragment includes a defective prophage under these conditions. It is of interest to know whether the phage genes of the lysogenic heterogenote are derived from the transducing particle or from the healthy phage.

The point has been tested only for the h locus. The results are given in Table 4. They show that this locus is derived from the nontransducing phage which was used at high multiplicity.

When such lysogenic heterogenotes lose the gal ${ }^{+}$character, the galsegregants remain lysogenic, in agreement with the findings of Morse et al. (1956a).

\section{Transduction of Lysogenic Recipients}

When a lysogenic recipient is used, according to Weigle (1957), the majority of transduction clones are composed of cells lysogenic for the phage originally carried, not for the superinfecting phage from the transducing lysate. The labor of examining a large number of such clones for carried phage is reduced by using HFT lysates of $\lambda \mathrm{h}$ and recipients carrying $\lambda \mathrm{h}^{+}$. Spot testing of purified isolates from different transduction clones then enables us to verify the results of Weigle for the h locus; and to select for further study the rare cases where this locus behaves exceptionally. Table 5 shows that the acquisition of the $\mathrm{h}$ allele may result either from substitution ${ }^{3}$ or from double lysogenization.

3 The data of Table 6 rule out the possibility that the substitutions are segregants from doubly lysogenic cells. Out of $216 \mathrm{gal}^{+}$subcolonies picked after more extensive growth than that involved in the isolation procedure of Table 5, not one is singly lysogenic for $\mathrm{h}$. 
TABLL: 5

Transdeteton of lysogene Strains

\begin{tabular}{ccc}
\hline Recipient & $\begin{array}{c}\text { Transtucing } \\
(\mathrm{HFT})\end{array}$ & $\begin{array}{c}\text { Phage type of transduction } \\
\text { clones }\end{array}$ \\
Strain $112\left(\lambda \mathrm{h}^{+}\right)$ & $\lambda \mathrm{h}$ & 112 are $\left(\lambda \mathrm{h}^{+}\right)$ \\
& 3 are $\left(\lambda \mathrm{h}^{+}\right)(\lambda \mathrm{h})$ \\
& $1 \mathrm{is}\left(\lambda \mathrm{h}^{+}\right.$defective $)$ \\
Strain $112\left(\lambda \mathrm{m}_{5} \mathrm{cos}\right)$ & 87 are $\left(\lambda \mathrm{h}^{+}\right)$ \\
& $4 \mathrm{are}(\lambda \mathrm{h})\left(\lambda \mathrm{m}_{5} \mathrm{cos}_{2}\right)$ \\
& 3 are $(\lambda \mathrm{h})$ \\
\hline
\end{tabular}

${ }^{a}$ Infection was as in Table 1 , except that a lower cell density $\left(1 \times 10^{\varnothing}\right)$ was used. The input multiplicity was $10-20$; adsorption was not measured. Gal ${ }^{+}$ papillae were restreaked, and $\mathrm{gal}^{+}$subcolonies were tested for carried phage. The defective strain liberated on induction about $10^{2}$ plaques of $\lambda \mathrm{h}^{+}$per milliliter, under conditions where lysogenic strains liberate about $10^{10}$. The $\left(\lambda \mathrm{h}^{+}\right)$ strains were scored by spot testing on CR63 and C600. They were not examined further.

\section{Correlated Losses of Prophage Alleles and the Exogenes}

All of the doubly lysogenic strains were heterogenotes. They were studicd further to see if there was any correlation between loss of the $\mathrm{gal}^{+}$character and changes in prophage content.

Three different methods were employed. (1) Unselected substrains from a doubly lysogenic heterogenote were examined for prophage con tent and for the gal character. (2) Gal ${ }^{-}$substrains were examined for prophage content. (3) Substrains which had lost the $\mathrm{h}$ prophage allele were examined for prophage content and for the gal character.

The experimental design in each case insured that each substrain tested was of independent origin; i.e., that the closest common ancestor of any two substrains was a cell of the original type. This was accomplished as follows:

(1) Independent unselected colonies were picked in two different ways. In both cases, one started by streaking a young colony onto tryptone agar.

(a) In the first series, 151 colonies from such a plating were restreaked. One subcolony from each was tested. If the subcolony was not $112(\lambda \mathrm{h})$ $\left(\lambda \mathrm{m}_{5} \mathrm{CO}_{2}\right)$ and $\mathrm{gal}^{+}$, a second subcolony from the same plate was tested. In sixteen cases, both subcolonies were different from the original type. They were discarded. The remaining changed types were recorded. They are of completely independent origin. Some of those discarded may also 
have been of independent origin; the number of unchanged subcolonies was therefore corrected from 109 to $84 .^{4}$

(b) In the second series, 200 colonies were tested on galactose agar, and spot tested on CR63. Only those colonies which were galactose positive and produced a spot on CR63) were restreaked. From each of these a single subcolony was picked and tested. Here our estimate of the unchanged types will be correct. The data are not completely independent because the screening procedure does not distinguish $112(\lambda \mathrm{h})$ $\left(\lambda \mathrm{m}_{5} \mathrm{CO}_{2}\right)$ from $112(\lambda \mathrm{h})\left(\lambda \mathrm{m}_{5}\right), 112(\lambda \mathrm{h})$, ete; but the results show that the frequency of the latter types among gal ${ }^{+}$cells is very small.

(2) 'lo select gal- subcolonies, a young colony was first streaked onto galactose agar. From this plate, a number of $\mathrm{gal}^{+}$colonies were restreaked. One then selected from each plate one gal $^{-}$subcolony and one gal ${ }^{+}$subcolony as control and tested them for carried phage. If the prophage content of the gal ${ }^{+}$subcolony differed from that of the original strain, the negative was not tabulated in Table 7.

(3) If selection was for the $\mathrm{h}$ character, many colonies from the initial streak plate were each suspended in broth and diluted to give 50-100 colonies per plate (on tryptone agar). After incubation, the plates were replicatcd onto a 1:1 mixture of $\mathrm{C} 600$ and $\mathrm{CR} 63$. The replicas were then irradiated with ultraviolet light. Subcolonies no longer carrying the $h$ allele gave turbid spots on the replicas, whereas those carring the $h$ allele produced clear spots. From each plate, one colony with, and one without the $h$ allele were tested. If the colony with the $h$ allele was not identical with the original strain, the corresponding $\mathbf{h}^{+}$colony was not used in compiling Table 7.

The results of the three procedures are shown in Tables 6 and 7 . In Table 7 are listed all independent losses of $\mathrm{gal}^{+}$or of $\mathrm{h}$, including those shown in Table 6 . Besides the colonies of Table 6, a total of 234 pairs of subcolonies were tested in obtaining the data of Table 7 . Table 8 shows the total data from these pairs, including losses which may not be independent, but excluding one experiment in which more extensive growth had been allowed after the initial streaking.

Tables 6 and 7 show that a variety of events are possible, but that the

${ }_{4}$ The ratio of changed to unchanged types has no absolute significance because it varies from 0 to $\infty$ as growth proceeds. However, we wish to use (for comparison within a single experiment) the frequency of $\mathrm{gal}^{-}$subcolonies within each group of subcolonies identical in prophage constitution. An accurate measurement of the proportion of unchunged subcolonies is necessary for such comparisons. 
TABLE' 6

Indep'endent Unselected Clones ${ }^{a}$

\begin{tabular}{|c|c|c|c|c|c|c|c|}
\hline \multirow{2}{*}{ Phage type } & \multicolumn{2}{|c|}{ Series 1} & \multicolumn{2}{|c|}{ Series II } & \multicolumn{2}{|c|}{ Total } & \multirow{2}{*}{ Total } \\
\hline & $\mathrm{Gal}^{+}$ & $\mathrm{Gal}^{-}$ & $\mathrm{Gal}^{+}$ & $\mathrm{Gal}^{-}$ & $\mathrm{Gal}^{+}$ & Gal $^{-}$ & \\
\hline$(\mathrm{h})\left(\mathrm{m}_{5} \mathrm{CO}_{2}\right)$ & $(84)$ & 0 & $(105)$ & 2 & (189) & 2 & (191) \\
\hline "Rearrangement" & 0 & 0 & 1 & 3 & 1 & 3 & 4 \\
\hline$(\mathrm{h})\left(\mathbf{m}_{5}\right)$ & 0 & 1 & 4 & 2 & 4 & 3 & 7 \\
\hline$\left(\mathrm{m}_{5}\right)\left(\mathrm{m}_{5} \mathrm{co}_{2}\right)$ & 5 & 0 & 7 & 2 & 12 & 2 & 14 \\
\hline$\left(\mathrm{m}_{5} \mathrm{co}_{2}\right)$ & 6 & 11 & 3 & 12 & 9 & 23 & 32 \\
\hline (b) & 0 & 1 & 0 & 1 & 0 & 2 & 2 \\
\hline$\left(m_{0}\right)$ & 0 & 2 & 0 & 8 & 0 & 10 & 10 \\
\hline$\left(\mathrm{m}_{3} \mathrm{hCO}\right)$ & 0 & 0 & 1 & 0 & 1 & 0 & 1 \\
\hline \multirow[t]{2}{*}{ Discarded } & $7^{b}$ & $9^{b}$ & 0 & 0 & - & - & \\
\hline & 95 & 15 & 121 & 30 & 216 & 45 & 261 \\
\hline
\end{tabular}

"Details in text.

${ }^{\iota}$ Not included in totals.

TABLE 7

INDEpendent Loss Events From DOUBLy I,Ysogesic Strainsa

\begin{tabular}{|c|c|c|c|}
\hline \multirow{2}{*}{ Carried prophage (s) } & \multirow{2}{*}{$\frac{\text { Selected for loss of } \mathrm{gai}^{+}}{\left(\mathrm{gal}^{-}\right)}$} & \multicolumn{2}{|c|}{ Selected for loss of $h$} \\
\hline & & $\left(\mathrm{gal}^{+}\right)$ & $\left(\mathrm{gal}^{-}\right)$ \\
\hline (h) $\left(\mathrm{m}_{5} \mathrm{CO}_{2}\right)$ & 20 & & \\
\hline "Rearrangement" & 9 & & \\
\hline (h) $\left(\mathrm{heO}_{2}\right)$ & 1 & & \\
\hline (h) $\left(m_{5}\right)$ & 7 & & \\
\hline$\left(m_{i}\right)\left(m_{b} \cos _{2}\right)$ & 11 & 22 & 20 \\
\hline$\left(\mathrm{m}_{5} \mathrm{CO}_{2}\right)$ & 71 & 15 & 44 \\
\hline (h) & 6 & & \\
\hline$\left(\mathrm{m}_{5}\right)$ & 14 & 1 & 16 \\
\hline \multirow[t]{3}{*}{$\left(m_{5} h\right)$} & 1 & & \\
\hline & - & - & - \\
\hline & 140 & 38 & 80 \\
\hline
\end{tabular}

${ }^{a}$ Details in text.

most frequent is the joint loss of the exogenes and the entire (h) prophage. This suggests that the two structures are associated with each other.

This is not the only interpretation possible. One might imagine, for example, that the exogenes were located sufficiently close to either prophage so that the loss of gal $^{+}$(imagined to be a recombinational event) 
occurred at the time of prophage pairing but was sufficiently far from the markers used so that it exhibited no linkage with them. Changes in prophage content and loss of $\mathrm{gal}^{+}$would then be correlated because prophage pairing would be prerequisite to both. One would expect that the distribution of change events would be the same among the cells which become negative as among those which remain positive, but that the proportion of cells changed in prophage content would be greater among the former.

The data are adequate to test this hypothesis. The distribution of changed types among the gal- substrains is significantly different from that among the gal ${ }^{+}$substrains. Even if one corrects for the fact that the few substrains which may have undergone more than one change event might be more common among the negatives than among the positives, there is still an excess of the type $112\left(\mathrm{\lambda} \mathrm{m}_{5} \mathrm{CO}_{2}\right)$ among the negatives.

Whereas such a general correlation between loss of gal ${ }^{+}$and changes in prophage content cannot account for all the data, there is reason to believe it explains some of it. This is indicated by the fact that for every type of change in prophage content of which there is more than one instance, the frequency of gal- substrains is higher among the changed substrains than among those which are unchanged (Table 6). For most types, this is significant at the $5 \%$ level. Particularly striking are such

TABI.E: 8

Total Frequency of Types (incleding Nonindependent Losses) in Data Used in Compiling TABLE $7^{a}$

\begin{tabular}{|c|c|c|c|c|}
\hline \multirow{2}{*}{ Carried prophage (s) } & \multicolumn{2}{|c|}{ Selected for h character } & \multicolumn{2}{|c|}{ Selected for gal character } \\
\hline & $\mathrm{Gal}^{+}$ & $\mathrm{Gal}^{-}$ & $\mathrm{Gal}^{+}$ & $\mathrm{Gal}^{-}$ \\
\hline (h) $\left(\mathrm{m}_{5} \mathrm{co}_{2}\right)$ & 67 & 7 & 75 & 20 \\
\hline "Rearrangement" & 1 & 1 & 1 & 5 \\
\hline (h) $\left(\mathrm{hCO}_{2}\right)$ & 2 & 1 & 1 & 2 \\
\hline$(h)\left(m_{5}\right)$ & 0 & 3 & 2 & 5 \\
\hline$\left(\mathrm{m}_{5}\right)\left(\mathrm{m}_{5} \mathrm{co}_{2}\right)$ & 15 & 21 & 9 & 11 \\
\hline (h) & 0 & 4 & 0 & 3 \\
\hline$\left(\mathrm{m}_{5} \mathrm{Co}_{2}\right)$ & 7 & 29 & 6 & 41 \\
\hline$\left(\mathrm{m}_{5}\right)$ & 2 & 8 & 0 & 4 \\
\hline
\end{tabular}

a In the experiments of Table 7 , a control colony was always picked from the same elone as that in which the loss event had occurred. It was selected on the basis of not having lost the " $\mathrm{h}$ " (column 1) or the "gal"" (column 3) marker. Only those clones in which the control colony was $(h)\left(\mathrm{m}_{5} \mathrm{Co}_{2}\right)$ ( $\left.\mathrm{gal}^{+}\right)$were used in compiling Table 7. 
types as $112(\lambda \mathrm{h})$ and $112\left(\lambda \mathrm{m}_{5}\right)$. The former, being singly lysogenic for the prophage which came from the HFT lysate, might be expected to have as high a frequency of gal ${ }^{+}$substrains as any other type; instead, all such substrains observed have been gal ${ }^{-}$.

\section{Coupling Relationships in Doubly Lysogenic Strains}

Those strains which are doubly lysogenic for prophages differing at more than one locus liberate not only phages of the two parental genotypes but also some recombinants. For example, $112(\lambda \mathrm{h})\left(\lambda \mathrm{m}_{5}\right)$ liberates a small number $(<5 \%)$ of phage which are $m_{5}{ }^{+} h^{+}$or $m_{5} h$. In general, the recombinants are in sufficiently small proportion so that there is no ambiguity in inferring coupling relationships in the prophages. The proportion, in the yield, of the two parental types varies considerably with factors such as age of culture, which have not been controlled very precisely.

The class labeled "rearrangement" liberated all eight possible phage types, but in different proportions than $112(\lambda \mathrm{h})\left(\lambda \mathrm{m}_{5} \mathrm{Co}_{2}\right)$. On mixed indicators it gives the same appearance as one would expect of a reciprocal recombinant $112(\lambda \mathrm{h})\left(\lambda \mathrm{m}_{5} \mathrm{CO}_{2}\right)$. However, on $\mathrm{C} 600, \mathrm{~m}_{5} \mathrm{CO}_{2}$ plaques are more common than $m_{5}$ plaques. A typical induced lysate had the following composition. Plated on mixed indicators: hco $\mathrm{O}_{2}-259 ; \mathrm{h}-112$ $\mathrm{m}_{5} \mathrm{hCO}_{2}-8 ; \mathrm{m}_{5} \mathrm{~h}-1 ; \mathrm{m}_{5}$ (or $\left.\mathrm{m}_{5} \mathrm{CO}_{2}\right)-170 ;+\left(\right.$ or $\left.\mathrm{CO}_{2}\right)-22$. Plated on C600: $\mathrm{CO}_{2}\left(\right.$ or heO $\left.\mathrm{O}_{2}\right)-274,+($ or $\mathrm{h})-106, \mathrm{~m}_{5} \mathrm{CO}_{2}\left(\right.$ or $\left.\mathrm{m}_{5} \mathrm{hCO}_{2}\right)-147, \mathrm{~m}_{5}$ (or $\left.\mathrm{m}_{5} \mathrm{~h}\right)-45$.

This is the only class which we have distinguished on the basis of proportion of liberated types rather than their qualitative presence or absence. There may well be variations in ratios of liberation within other classes; so one might question the consistency of separating this group from $112(\lambda \mathrm{h})\left(\lambda \mathrm{m}_{5} \mathrm{co}_{2}\right)$. However, it is strikingly and obviously different from the original type, a continuous spectrum of intermediates has not been observed, and it would seem foolish therefore to ignore its existence.

\section{Rate of Loss of the h Prophage Allete}

In those experiments in which subcolonies were selected for loss of the $h$ allele, data were incidentally accumulated on the rate of loss of this allele. From the number of colonies giving clear or turbid spots on the replica, one can calculate the rate of loss to be $6.6 \times 10^{-3}$ per bacterium per generation. Three experiments with the same strain gave ralues 6.3 , 6.5 , and $6.8 \times 10^{-2}$, respectively. In one experiment with a doubly 
lysogenic strain $112(\lambda)(\lambda \mathrm{h})$ from the first series, the figure was $3.0 \times$ $10^{-3}$. The rates of other change events can be calculated from this one and from the data of Tables 6 and 7 .

Relationship of the Exogenes to the Prophages in a Derived Doubly Lysogenic Strain

The types $112(\lambda \mathrm{h})\left(\lambda \mathrm{m}_{5}\right), 112(\lambda \mathrm{h})\left(\lambda \mathrm{hco}_{2}\right)$, and $112\left(\lambda \mathrm{m}_{5}\right)\left(\lambda \mathrm{m}_{5} \mathrm{co}_{2}\right)$ may be interpreted as changes in one of the two prophages, the other prophage remaining unchanged. Obviously, this is not the only possibility; and it is desirable to test this hypothesis as rigorously as we can. One way is to examine further prophage changes. The results of an analysis of one such strain are given in Table 9 . Within the precision of the statistics given, the $(h)$ and $\left(m_{5}\right)$ prophages must be considered to bear, respectively, the same relationship to the exogenes as the (h) and $\left(\mathrm{m}_{5} \mathrm{CO}_{2}\right)$ prophages did in the original strain.

It is important to realize that the analysis of $112(\lambda \mathrm{h})\left(\lambda \mathrm{m}_{5} \mathrm{co}_{2}\right) \mathrm{com}$ prises entirely measurements on one doubly lysogenic strain. It is always hazardous to generalize from a single instance. The segregation patterns of the three strains $112(\lambda \mathrm{h})\left(\lambda \mathrm{h}^{+}\right)$of Table 5 were examined less extensively and conformed to the same pattern. One other strain of the type $112(\lambda \mathrm{h})\left(\mathrm{\lambda} \mathrm{m}_{5}\right)$ likewise corroborates the results of Table 9.

TABLE 9

IndePendent Unselatected Clones from $112(\lambda \mathrm{h})\left(\lambda \mathrm{m}_{5}\right)$ HeTEROGENOTE ${ }^{a}$

\begin{tabular}{|c|c|c|c|c|c|c|}
\hline \multirow{2}{*}{ Carried phage(s) } & \multicolumn{3}{|c|}{$112(\lambda \mathrm{h})\left(\lambda \mathrm{m}_{\mathrm{s}}\right)$} & \multicolumn{3}{|c|}{$112(\lambda \mathrm{h})\left(\lambda \mathrm{m}_{5} \mathrm{CO}_{2}\right)$} \\
\hline & $\mathrm{Ga}^{+}$ & $\mathrm{Gal}^{-}$ & Total & $\mathrm{Gal}^{+}$ & $\mathrm{Gal}^{-}$ & Total \\
\hline$(\mathrm{h})\left(\mathrm{m}_{5}\right)$ & 112 & 15 & 127 & 194 & 8 & 202 \\
\hline$\left(\mathrm{m}_{\tilde{n}}\right)$ & 9 & 26 & 35 & 21 & 35 & 56 \\
\hline (h) & 0 & 2 & 2 & 0 & 2 & 2 \\
\hline \multirow[t]{3}{*}{$\left(m_{5} h\right)$} & 0 & 0 & 0 & 1 & 0 & 1 \\
\hline & - & - & - - & -- & $-\cdots$ & $\ldots$ \\
\hline & 121 & 43 & 164 & 216 & 45 & 261 \\
\hline
\end{tabular}

a Jetails as in Table 6 , except starting culture was $112\left(\lambda h_{1}\right)\left(\lambda m_{5}\right)$ heterogenote which had arisen spontaneously from $112(\lambda \mathrm{h})\left(\lambda \mathrm{m}_{5} \mathrm{co}_{2}\right)$. On the right, the data of Table 6 are reorganized by lumping those classes which would be indistinguishable if the $\mathrm{CO}_{2}$ locus were not scored for. The amount of growth during which change events could occur was about the same in the two series. The two sets of numbers are not significantly different. 


\section{I)ISCUSSION}

Table 10 summarizes present knowledge concerning transduction and segregation of the gal region.

\section{Transductional Events}

In constructing a model for a complex phenomenon, it is well to start from the simplest event. In this system, that event would seem to be the transduction of a sensitive recipient at low multiplicity. The most frequent result is the production of a defectively lysogenic heterogenote which generally loses the gal ${ }^{+}$character and the immunity to superinfection simultaneously. Sensitives stable for the galactose character also orcur. Lyssogenics are vanishingly few.

One can therefore entertain two possibilities for the nature of the transducing particle. (1) It contains a defective phage genome attached

TABLE 10

Thansductional and Seghegational Even'ts

\begin{tabular}{|c|c|c|c|}
\hline Recipient cell & Multiplicity of HFT lysate & Transduction clone & $\mathrm{Gal}^{-}$derivatives \\
\hline Sensitive & $\begin{array}{l}\text { High } \\
\text { Low ( }+ \text { high multi- } \\
\text { plicity of non- } \\
\text { transducing } \\
\text { phage) }\end{array}$ & $\begin{array}{l}\text { Defective lysogenic } \\
\text { RARli: Sensitive, } \\
\text { stable } \\
\text { Lysogenic } \\
\text { Lysogenic } \\
\text { Lyrogenic for (hal- } \\
\text { lele from) non- } \\
\text { transducing } \\
\text { phage }\end{array}$ & $\begin{array}{l}\text { Sensitive } \\
\text { RARti: Defective } \\
\text { lysogenie } \\
\qquad- \\
\text { Lysogenic } \\
\text { Lysogenic } \\
\text { Lysogenic }\end{array}$ \\
\hline Lysogenic & No effect & $\begin{array}{l}\text { Lysogenic for (hal- } \\
\text { lele from) car- } \\
\text { ried phage } \\
\text { RARE: Doubly } \\
\text { lysogenic } \\
\text { Lysogenic for } \\
\text { superinfecting } \\
\text { phage }\end{array}$ & $\begin{array}{l}\text { Lysogenic } \\
\text { Prophage constitu- } \\
\text { tion generally } \\
\text { changed } \\
\text { Lysogenic }\end{array}$ \\
\hline
\end{tabular}

"Morse (personal communication) has found that about $10 \%$ of the galsegregants from heterogenotes which are defective lysogenics are homogenotes which are defective lysogenics. 
to the exogenes. The HFT lysates would be presumed to be mixtures of normal, nontransducing phages and such transducing defectives. (2) The lysate contains only one type of phage particle with two mutually exclusive alternatives on single infection (other than lysis): (a) transduction, to yield a defective prophage and (b) lysogenization, to yield a normal prophage.

No experiments yet performed really distinguish between these alternatives. The impressive feature of the present data, however, is that there is no necessity for supposing that the same infecting particle ever both transduces and gives rise to a nondefective prophage. We shall tentatively adopt possibility (1), because it makes some of our data more readily interpretable.

Now we can consider the more complicated situations:

(a) Transduction of a sensitive at high multiplicity. From fig. 1 we are led to believe that more than one particle is important. Table 1 shows that the only multiparticle effect we need postulate involves a single transducing particle and one or more other phage particles, which need not be able to transduce. It is in every way more informative, therefore, to consider the case of mixed infection with an HFT lysate at low multiplicity and a nontransducing lysatc at high multiplicity.

In this case, almost all transduction clones are composed of cells lysogenic for the $\mathrm{h}$ allele of the nontransducing phage (Table 4). When the transduced region is lost, the prophage is not lost with it. If one transduces sensitive cells at low multiplicity with the lysate from the new lysogenic heterogenote, one again gets a majority of defectives.

These facts are readily understandable on the basis of possibility (1) above. The lysogenic heterogenote may be imagined to carry both a healthy prophage and a defective prophage, the latter comprising part of the exogenotic fragment. Iittle is known about the nature of the prophage defect. There is therefore no reason to assume that the transducing particle carries a copy of the $h$ gene at all. We can explain Table 4 by assuming that it does not.

(b) Transduction of a lysogenic recipient. The efficiency of transduction is constant at approximately the level for sensitive recipients transduced at low multiplicity. One may assume that the specific immunity of the lysogenic cell prevents the action of healthy phage in increasing the efficiency of transduction. A single particle then transduces in presumably the same manner that it would a sensitive. A sensitive recipient would now carry a defective prophage. The lysogenic 
recipient carries its original prophage, perhaps in addition to a defective prophage. There is again no evidence that the defective prophage contains an $h$ gene.

The double lysogenizations and prophage substitutions can be viewed as due to either (a) coincidental events involving phage particles other than transducing particles; or (b) occasional transductions by particles which give normal prophages. The former interpretation is simpler and should be accepted until proven untenable. The doubly lysogenic strains, therefore, may well carry not only two prophages, but also a defective prophage attached to the gal region.

\section{Segregational Events}

From the results of Tables 6 and 7 , we concluded that the exogenes and the $(\mathrm{h})$ prophage were probably attached in the strains studied. This does not tell us whether they entered the cell as a unit or, as suggested in the previous paragraph, separately. Results similar to those of Table 2 are obtained if one transduces sensilives at low multiplicities with the lysates produced by induction of thedoubly lysogenic heterogenotes. Therefore, a defective prophage may be present which bears the same relationship to the exogenes as it would in a singly lysogenic or defeetively lysogenic heterogenote. How this whole structure of defective prophage and gal genes may be associated with the (h) prophage is an open question. These studies have not revealed any obvious linkage between the exogenes and any of the prophage markers used.

Two possibilities for segregational events from strains doubly lysogenic for $\lambda$ were listed by Appleyard (1954): (a) They are entirely recombinational, all apparently singly lysogenic derivatives being really double and "homozygous." (b) There is a separate mechanism by which a prophage can actually be lost completely. The only feature of the present data which bears on this point is the several oceurrences of types which are singly lysogenic for a recombinant. The rare recombinants $m_{5} h$ and $\mathrm{m}_{5} \mathrm{hcO}_{2}$ have occurred only as singles. This is unlikely to happen with a simple recombinational mechanism, and does not occur with strains doubly lysogenic for P2 (Bertani, 1956).

For the same reason, however, the "separate mechanism" is very unlikely to be a simple failure of one of the prophages to replicate or to disjoin properly; such a process should not give rise to recombinants. These recombinants cannot be quantitatively accounted for as secondary events from any of the other types listed in Table 6 . It is therefore 
likely that prophage pairing is a prerequisite both for recombination and for loss.

It is of interest, also, that reciprocal recombinants have not occurred in equal numbers. The type $\left(\mathrm{m}_{5}\right)$ is more common than $\left(\right.$ hco $\left.\mathrm{O}_{2}\right)$, regardless of whether the cell becomes singly lysogenic or retains one of the original prophages.

Existing data, including those presented here, are insufficient to give a general picture of the segregation patterns of strains doubly lysogenic. for $\lambda$. For such a picture we will need data from heterogenotes produced in the reciprocal manner and from double lysogenics which are not heterogenotes, and data with different markers. 'The high rate of production of changed types is similar to that observed previously for strains doubly lysogenic for $\lambda$ (Appleyard, 1954). Much more stable doubles have been found among the lysogenic survivors of co-operation experiments between different clear mutants (Kaiser, 1957); but since no singly lysogenic segregants were observed at all, the possibility is not excluded that loss of one prophage from such a strain induces lysis, which would make the apparent stability unreal.

It must be mentioned that there is one group of experiments for which the deta are not homogencous with the rest. This is the set of clones in Table 7 which were chosen as losses of h. The ratio of 112 $\left(\lambda \mathrm{m}_{5}\right)\left(\lambda \mathrm{m}_{5} \mathrm{co}_{2}\right)$ to $112\left(\lambda \mathrm{m}_{5} \mathrm{co}_{2}\right)$ is significantly higher than in Table 6 ; and its ratio among gal- cells is significantly higher than among the other cells of Table 7 which were selected as gal- ${ }^{-}$The reasons for this difference are not known. Some of the $112\left(\lambda \mathrm{m}_{5}\right)\left(\lambda \mathrm{m}_{5} \mathrm{Co}_{2}\right)$ clones of this group liberated a small ratio $(<1: 10)$ of $\mathrm{m}_{5}$ to $\mathrm{m}_{5} \mathrm{Co}_{2}$. This was not commonly observed in the other series.

In Table 10, we have been careful to avoid equating the $h$ allele with the whole prophage genome. Subsequent data, both of our own and of another group (Arber et al., 1957) have demonstrated the importance of making this distinction.

Arber et al. have superinfected the defective lysogenic strains obtained by transduction at low multiplicity with genetically marked phages, and have examined the genotypes appearing in the phage yield. They find that the genes $c, p_{4}$, and (rarely) $m_{6}$ are recoverable, but that $h$ is not. We have found that if strain $112\left(\lambda \mathrm{h}\left(\mathrm{o}_{2}\right)\right.$ is infected with an HFT lysate of $\lambda++$, the majority of the transduced isolates yield on lysis a mixture of $\lambda \mathrm{h}$ and $\lambda \mathrm{hco}_{2}$. Similarly, mixed infection of strain 112 by an HIFT lysate of phage A at low multiplicity, and a nontransducing lysatc 
of phage $B$ at high multiplicity yields transduced strains which contain all the prophage genes of phage $B$ and those from the $c$ region of phage $A$, but not the $h$ gene from phage $A$.

The data are most easily explained by assuming that the transducing particle carries the galactose region of the bacterium instead of a portion of its own genetic material, which includes the $h$ locus.

\section{ACKNOWLEDGMENTS}

The author wishes to thank Jrs. A. D. Kaiser, E. M. Lederberg, M. L. Morse, and $\mathbf{J}$, J. Weigle for eultures and phage stocks; Drs. G. Bertani and A. D. Kaiser for helpful discussion and advice; Drs. W. Arber, M. L. Morse, and J. J. Weigle for access to unpublished data; and Mrs. Anita Krivis for technical assistance.

\section{REFERENCES}

AppleYaRD, R. K. 1954. Segregation of new lysogenic types during growth of a doubly lysogenic strain derived from Escherichia coli $K-12$. Genetics 39, 440452.

Appleyard, R. K., McGregor, J. F., and Barrd, K. M. 1956. Mutations to extended host range and the occurrence of phenotypic mixing in the temperate coliphage lambda. Virology 2, 565-574.

Arber, W., Kellenberger, G., and Weigle, J. 1957. La defectuosité du phage lambda transducteur. Schweiz. Z. allgem. Pathol. u. Bakteriol. In press.

Bertani, G. 1956. The role of phage in bacterial genetics. Brookhaven Symposia in Biol. 8 (BNL 350(C-22)), 50-56.

Kaiser, A. 1. 1955. A genetic study of the temperate coliphage lambda. Virology $1,424-443$.

KaISER, A. D. 1957. Mutations in a temperate bacteriophage affecting its ability to lysogenize Escherichia coli. Virology 3, 42-61.

Morse, M. L., Lemerbera, E. M., and Lederbera, J. 1956a. Transduction in Escherichic coli $K-12$. Genetics 41, 142-156.

Morse, M. L., Leverberg, E. M., and Leoerberg, J. 1956b. Transductional heterogenotes in Escherichia coli. Genetics 41, 758-779.

WEIGLE, J. J. 1957. Transduction by coliphage $\lambda$ of the galactose marker. Virology $4,14-25$.

Wollaman, E. 1953. Sur le déterminisme génétique de la lysogénie. Ann. inst. Pasteur 84, 281-293. 